\title{
High Prevalence of Active Tuberculosis in Adults and Children with Idiopathic Inflammatory Myositis as Compared with Systemic Lupus Erythematosus in a Tuberculosis Endemic Country: Retrospective Data Review from a Tertiary Care Centre in India
}

\author{
Latika Gupta ${ }^{1}$ (D), Rohit Aggarwal ${ }^{2}$, Naveen $\mathbf{R}^{1}$ (D), Able Lawrence ${ }^{1}$ (D), Abhishek Zanwar ${ }^{1}$, Durga Prasanna \\ Misra $^{1}$ (D), Vikas Agarwal', Ramnath Misra' ${ }^{1}$ (D), Amita Aggarwal' ${ }^{1}$ (D) \\ 1Department of Clinical Immunology \& Rheumatology, Sanjay Gandhi Postgraduate Institute of Medical Sciences, \\ Lucknow, India, 'UPMC Myositis Centre, Division of Rheumatology and Clinical Immunology, University of Pittsburgh, \\ Pittsburgh, PA, United States of America
}

\section{ABSTRACT}

Aim: Infections are the leading cause of morbidity and mortality in idiopathic inflammatory myositis (IIM) with India being endemic for Tuberculosis (TB). We compared and contrasted the prevalence, clinical profile and outcomes of active TB in IIM with systemic lupus erythematosus (SLE). Methods: Medical records were reviewed for adults and children with IIM (Bohan and Peter criteria) and SLE (ACR criteria) at a tertiary care hospital in India from January 2015 to October 2017. Follow-up was recorded until February 2020 for all those who had developed active TB. Results: Of 167 (132 adults and 35 juvenile) IIM and 280 (131 adults and 149 juvenile) SLE, active TB occurred in 24 (14.4\%) IIM (22 (16.7\% of 132) adults; 2 (5.71\% of 35$)$ juvenile) and 18 (6.4\%) SLE [(8 (6.1\% of 131) adults; 10 (6.7\% of 149 ) juvenile, $\mathrm{p}$-value $<0.01$ ]. Patients with IIM had higher odds of developing TB as compared with SLE [OR 2.24 ( $\mathrm{Cl} 1.5-5.5), \mathrm{p}=0.007$ ]. The risk of developing active TB was 68-fold and 30.4-fold higher in patients with IIM and SLE, respectively, as compared with the general population. Extrapulmonary forms were more common (14/24). Nearly half developed TB during active IIM, at a glucocorticoid dose of 0.25 (0-1.5) mg/kg/day. Over a follow-up duration of 27 months (8-184), all were cured of TB, though prolonged course of anti-tuberculous treatment was required in $25 \%$, and five IIM relapsed during treatment. Conclusion: Patients with IIM have increased risk of active TB, with common extrapulmonary forms, slow response, and relapses during treatment.

Mediterr J Rheumatol 2020;32(2):134-42

https://doi.org/10.31138/mjr.32.2.134

\section{Corresponding Author:}

Latika Gupta

Assistant Professor, Department of

Clinical Immunology and Rheumatology

Sanjay Gandhi Post Graduate Institute

of Medical Sciences

Lucknow, Uttar Pradesh, 226014

Tel.: +91-7408605517

E-mail: drlatikagupta@gmail.com,

latika@sgpgi.ac.in

Article Submitted: 1 Jul 2020; Revised Form: 13 Oct 2020; Article Accepted: 25 Oct 2020; Available Online: 30 Jun 2021

Keywords: Myositis, lupus, tuberculosis, India, infections, dermatomyositis, glucocorticoids

\section{INTRODUCTION}

A diagnosis of Idiopathic Inflammatory Myopathies (IIM) entails significant morbidity, and, at times, even mortality. ${ }^{1}$ In the developing world, infections are the leading contributor to such 
morbidity. Tuberculosis (TB) is one such infection, that remains a particular challenge in this part of the world. The emergence of drug-resistant strains has only added to the problem. Polypharmacy, in the setting of chronic illnesses, further compounds the problem.

Recent estimates suggest the prevalence of TB in India to be 3.2 cases per thousand population. ${ }^{2}$ Patients with IIM often require high doses of glucocorticoids for long periods. Poor cough reflex from pharyngeal and respiratory muscle involvement and immobility results in poor handling of both aerosols and oral secretions. Moreover, some patients IIM also have underlying complement pathway defects, such as Mannose Binding Lectin deficiency which is reasonably common and confers a higher risk of TB. ${ }^{3,4}$ Children are more likely to harbour such inherited deficiencies. ${ }^{5}$

Over the last two decades, public health initiatives in India have attempted to address the issues of rising prevalence, treatment default, and the emergence of drug resistance in TB with partial success. ${ }^{2}$ On the same lines, there have been efforts towards decreasing the usage of glucocorticoids in rheumatic disorders too. The changing dynamics of therapeutic practices could have a bearing on the prevalence of tuberculosis in these diseases, and also influence the ways this problem is addressed. Since a diagnosis of IIM entail long term treatment with high doses of glucocorticoids, we sought to determine the prevalence of active TB in adults and children with IIM and compare it with patients diagnosed with Systemic Lupus Erythematosus (SLE), another rheumatic disease that requires high dose glucocorticoids and are associated with higher incidence and prevalence of TB. ${ }^{6,7}$

\section{METHODS}

Medical records from paper charts and electronic medical records were reviewed for adults and juvenile patients with definite and probable IIM by Bohan and Peter criteria 1975$)^{8}$ following up at a tertiary care hospital in India from January 2015 to February 2020. The approach was all-inclusive irrespective of a past history of TB. Radiographs of the chest are usually obtained in all patients before beginning immunosuppression, ruling out the possibility of active pulmonary TB at initiation of immunosuppressant (IS) therapy. Patients with drug-induced myopathies, inherited or degenerative myopathies and endocrine, infectious, or metabolic muscle disorders were excluded. Institute ethical clearance for waiver of consent for retrospective data review was obtained [2017-41-IP-76] as per local guidelines. ${ }^{9}$ Demographic variables and clinical profiles, as well as disease activity, were noted. Records of adults and children with SLE (ACR criteria 1997) collected for a previous study were drawn for comparison with IIM. ${ }^{10}$

Assuming a confidence interval of $95 \%$ and a margin of error $2 \%$ with a $1.8 \%$ prevalence of TB in IIM from an- other study, ${ }^{11}$ the sample size was calculated to be 170 . An event of TB was defined by either the demonstration of acid-fast bacilli (AFB) in the tissue or fluid obtained or presumptive diagnosis ${ }^{12}$ based on clinical and radiological features and improvement with anti-tuberculous therapy (ATT). Information on the organs involved, past or family history of TB, duration of ATT, drug interactions and adverse events, glucocorticoid and other immunosuppressive drugs received were obtained. A glucocorticoid (GC) dose $>0.5 \mathrm{mg} / \mathrm{kg}$ was defined as high dose steroids. Details on outcomes such as cure of TB, resistance, relapses of TB and the primary disease, and death were recorded.

Active disease and relapse of IIM were defined as per physician assessment- based on new/subacute muscle weakness alongside elevated muscle enzymes or weakness requiring an increase in immunosuppression as assessed by the treating physician. A worsening of skin rash was also recorded as flare of disease in those with dermatomyositis or overlap myositis. The presence of interstitial lung disease (ILD) was defined as the presence of lung fibrosis by high-resolution computed tomography and evidence of restrictive physiology on pulmonary function tests. Screening for ILD was done only in those with breathlessness on exertion or crackles on respiratory examination. Chronic liver disease defined as a progressive deterioration of liver functions for more than six months, which includes synthetic functions, detoxification and excretory functions, assessed using a combination of ultrasound liver, liver function tests, coagulation profile and elastography. ${ }^{13}$ Overlap myositis defined by presence of myositis and fulfilling one CTD criteria. ${ }^{14-18}$ Antisynthetase syndrome defined by Connor's criteria. ${ }^{19}$ Complete response, partial response and relapse in SLE as defined by EULAR/ERA-EDTA criteria. ${ }^{20}$

\section{STATISTICAL ANALYSIS}

Comparisons were drawn between SLE and IIM patients. Descriptive statistics and non-parametric tests were used for comparisons. Results are expressed as median $\pm 2 \mathrm{SD}$. The analysis was done using GraphPad Prism for Mac (trial version 7)

\section{RESULTS}

The IIM cohort had 132 adults and 35 juvenile IIM patients with mean age at diagnosis 32 and eight years respectively (Table 1). The SLE cohort had 131 adult and 149 juvenile patients with mean age at diagnosis 32.4 and 13.7 years. Among adult IIM patients $42.4 \%$ (56) were DM, $21.2 \%$ (28) PM, 9.8\% (13) anti-synthetase, and $26.5 \%$ (35) overlap myositis. Among juvenile IIM patients, most were JDM 74.3\% (26) (Table 1). 
Table 1. Population demographics of SLE and IIM cohorts.

\begin{tabular}{l|l|l|l|l}
\hline \multicolumn{1}{c|}{ Variable } & Adult SLE & Pediatric SLE & \multicolumn{1}{|c}{ Adult IIM } & \multicolumn{1}{c}{ Juvenile IIM } \\
\hline Patient number & 131 & 149 & $132^{*}$ & $35^{\#}$ \\
\hline $\begin{array}{l}\text { Median follow up at the time of inclusion into the } \\
\text { study (years) }\end{array}$ & 3.0 & 6.0 & 5.5 & 8.0 \\
\hline Female: Male & $8: 1$ & $9: 1$ & $6.3: 1$ & $1.5: 1$ \\
\hline Median age (years) & 32.4 & 13.7 & 32 & 8 \\
\hline Lupus nephritis (\%) & 51.9 & 100 & NA & NA \\
\hline Chronic kidney disease (\%) & 4.6 & 7.3 & 0 & 0 \\
\hline Number of TB cases & 8 & 10 & 22 & 2 \\
\hline Incidence rate/ 100 patient years follow up & 2.0 & 1.1 & 2.3 & 1.9 \\
\hline
\end{tabular}

*56 DM, 28 PM, 13 Anti-synthetase syndrome and 35 Overlap myositis; "26 jDM, 1 PM and 8 Overlap Myositis

The prevalence of tuberculosis in adults and children with IIM

Active TB was seen in 24 (14.4\%) IIM patients (Table 2) with 22 (16.7\%) being in adults and two (5.71\%) in juvenile IIM cases. Of those with TB, 18 had DM; three had PM, four anti-synthetase syndrome and seven had overlap myositis.

Lung was most common focus of TB, 10 patients had pulmonary TB and 2 patients had isolated pleural effusion. Extra-pulmonary TB was seen in 12 patients: Lymph node and disseminated TB was found in four patients each. Four patients had unusual site of involvement. Two had bone TB; one of them had pyomyositis in addition. One patient each had renal and gastrointestinal involvement (Figure 1).

TB occurred at median 2 (1-2.5) years after the onset of IIM in adults, and recurrence was seen in one of the 22 (4.5\%) cases 5.5 years after complete treatment. On the other hand, TB was diagnosed at the disease onset in one of the two cases of jDM, with a recurrence of MDR sputum positive pulmonary TB 4.5 years later.
Prevalence of tuberculosis in adults and children with SLE One-hundred thirty-one adults and 149 children with SLE were included for comparison. Of the 131 (8:1 female:male) adult SLE patients (median age 32.4 years, mean follow-up 3 years, 68 (51.9) had lupus nephritis and 4.6 had chronic kidney disease) eight developed Tuberculosis (6.10\%).

Half the patients developed TB within 2 years and a half were extra-pulmonary (1 lymph node, 1 peritoneal, 1 bone, 1 CNS). Of the 149 (female:male 9:1) paediatric SLE patients (median age 13.7 years, mean follow up 6 years, all had lupus nephritis) and 10 (6.71\%) developed TB. Six of 10 had TB within six months of SLE diagnosis and half were extra-pulmonary (2 pericardial, 1 lymph node and 2 disseminated). One of them died of disseminated TB (Figure 1).

\section{Comparison of SLE and IIM patients with TB}

The overall incidence of TB in IIM was $14.37 / 100$ patients (2/100 patient years) and in SLE was 6.42/100 patients (2.386/100 patient years). The incidence of TB in adult IIM patient was 16.6/100 patients while in juvenile IIM
A. Sites of Tuberculosis

Lupus

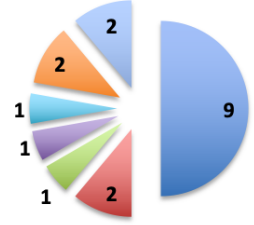

Myositis

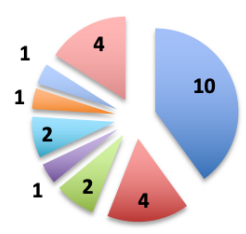

- Pulmonary

Lymph Node

Bone

- Gastrointestinal

- Pleural

Pyomyositis

- Granulomatous Interstitial Nphritis

$₫$ Disseminated
B. Prevalence of Tuberculosis

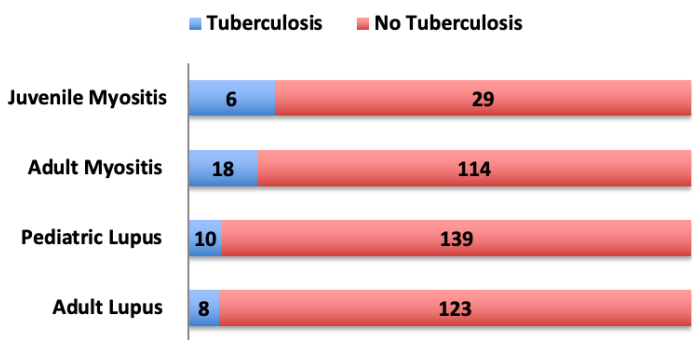

Figure 1. Sites of tuberculosis in SLE and IIM 1b. Prevalence of tuberculosis in SLE and IIM. 
Table 2. Clinical profile of patients with Tuberculosis.

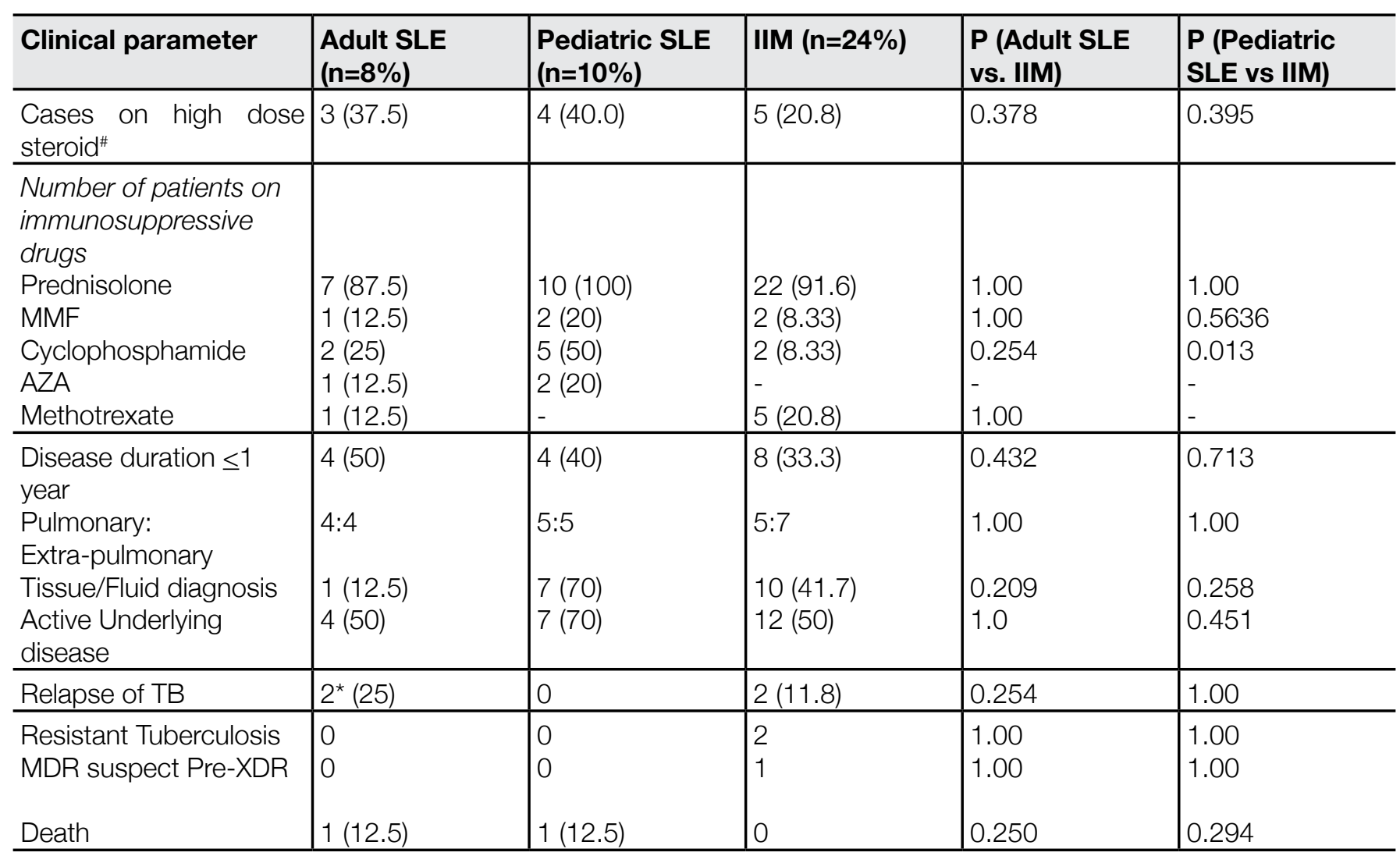

${ }^{\#}(>0.5 \mathrm{mg} / \mathrm{kg}){ }^{*}$ One patient each had CKD and family history of TB.

SLE: systemic lupus erythematosus; IIM: idiopathic inflammatory myopathies; MDR: multidrug resistant; XDR: extreme drug resistance; TB: tuberculosis.

$P$ calculated using Fisher's exact between groups.

was 5.71/100 patients. In SLE patients, incidence of TB is $6.1 / 100$ patients in adults and 6.7/100 patients in children. Considering an annual TB rate of 211 per 100,000 of the general population, ${ }^{2}$ the risk of developing active TB was 68-fold higher in patients with IIM and 30.4-fold higher in those with SLE. Patients with IIM had higher odds of developing TB as compared with SLE [odds ratio 2.24 (Cl 1.5-5.47), $\mathrm{p}=0.007$ ).

The was no difference in the age, duration from disease onset to development of tuberculosis, the prevalence of extra-pulmonary TB or death due to TB between IIM and SLE (Table 2). Family history of TB was present in two children and four adults with SLE.

\section{Factors predicting TB}

The IIM patients who developed TB were no different from those without TB with regard to the gender, type of IIM, or the age of onset of IIM (childhood versus adulthood). Most patients with IIM who developed TB $[\mathrm{n}=16(66.7 \%)]$ developed various other infections as well (Table 2). Three of them had opportunistic infections, one each with cytomegalovirus, esophageal candidiasis, and herpes zoster. Eight patients (33.3\%) had other risk factors for infections (ILD, Chronic Liver Disease, and pregnancy).

\section{Diagnosis of TB in IIM}

In almost half the cases $(n=13)$, the diagnosis was based on definite evidence of mycobacterial infection, i.e. smear evidence of acid-fast bacilli $(n=7)$, histology $(n=4)$, or growth in cultures ( $n=3)$ (Table 3). An empiric diagnosis was made in 11 patients. One patient was suspected to have miliary TB, but died before the diagnosis could be confirmed. One patient of DM had culture-proven pre-extremely drug-resistant (Pre-XDR) infection. She improved on therapy but suffered kanamycin induced hearing loss. IIM patients who developed TB had a median disease duration of $2(0.2-12)$ years.

\section{Diagnosis of TB in SLE}

The diagnosis of TB was empiric in most cases with adult SLE while in seven paediatric lupus it was based 
\begin{tabular}{l|l} 
MEDITERRANEAN JOURNAL & 32 \\
OF RHEUMATOLOGY & 2021
\end{tabular}

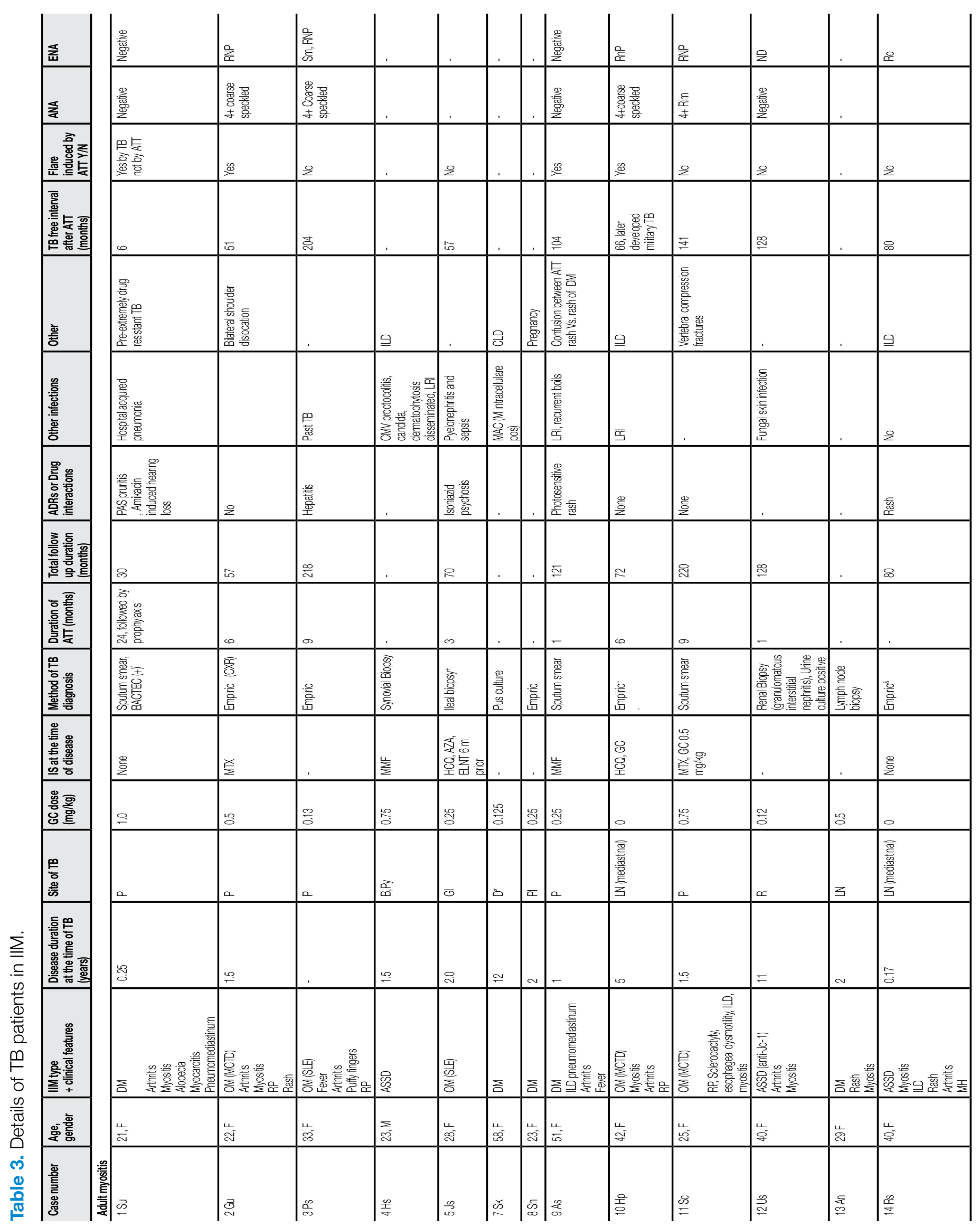



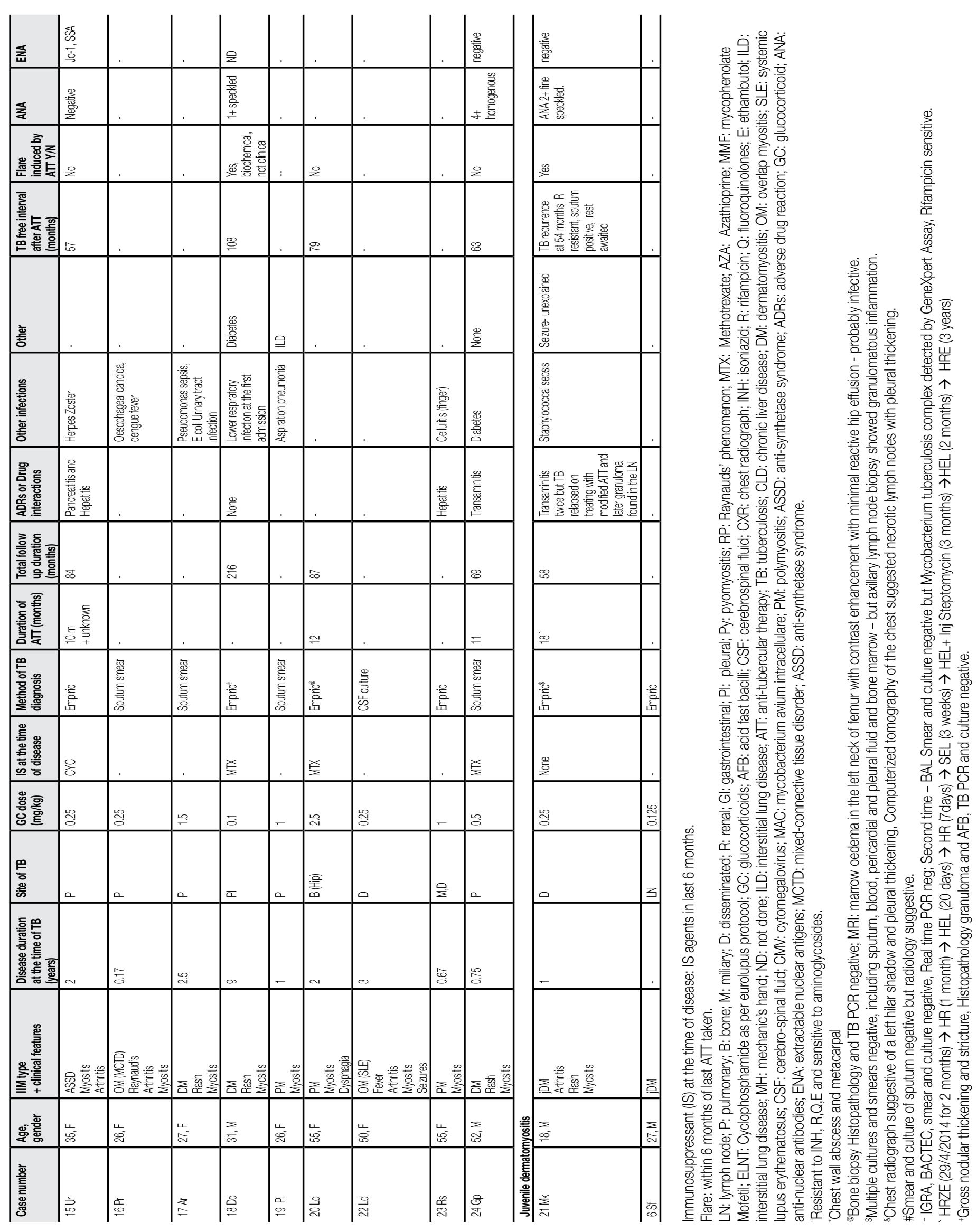
on tissue/smear positivity (Table 1). None of them was suspected to be drug resistant.

\section{The course of TB in IIM}

Fourteen were followed up after the diagnosis of TB, of which one was lost after one month of treatment. The median GC dose was $0.25(0.1-1.8) \mathrm{mg} / \mathrm{kg} /$ day at the time of diagnosis of TB. Nine of the 13 followed up (69.2\%) had 10 adverse events related to ATT: 5 had drug-induced hepatitis, one had pancreatitis, 2 had a skin rash; one of them was related to para-amino salicylate sodium (PAS) (Two patients had more than one adverse effect).

Median follow-up duration after the diagnosis of TB was 82 (69.3-126.3) months, while median TB free interval was 72.5 (57-107) months. The median duration of ATT was 9 months (5.3-11.2). A resolution of TB was documented in all who followed up. Two patients had a recurrence of TB, at 66 and 54 months respectively, despite taking a complete course of ATT. One of them developed military TB and the other sputum positive resistant TB. There was one death from TB in this cohort. A prolonged course of ATT was required in $25 \%$ of patients as compared with $2 \%$ in the general population. Confirmed drug resistance was 3\%, similar to the general population.

The treatment with ATT resulted in a relapse of myositis in 5 cases, of which flare of muscle disease was seen in 3 patients, while 2 had cutaneous involvement. However, by the last follow up visit, all patients had attained remission.

\section{The course of Tuberculosis in SLE}

Out of 18 cases of TB in patients with SLE, 10 were in remission, five had relapse of SLE during or after completion of ATT and 3 had persistent active disease. Among these two were lost to follow up, 14 were cured of TB and two died. Two out of 290 SLE patients (714 per 100000 patients) suffered mortality, as compared with 32 per 100000 rates in the general population. ${ }^{2}$ SLE patients with tuberculosis suffered 22 times higher mortality than that seen from TB in the general population.

\section{DISCUSSION}

Idiopathic inflammatory myositis is known to be a debilitating disease with a high risk of infections. We have previously described infections as the leading cause of in-hospital mortality in patients with IIM. ${ }^{1}$ Of the various infections, Tuberculosis is one that remains a particular challenge in India. Apart from being a Tuberculosis endemic zone, the emergence of drug-resistant strains has further compounded the picture in this region.

We found that $14 \%$ of patients with IIM developed $\mathrm{TB}$, and this risk was 68 times higher than the general population, and 2.24 times higher than SLE patients. Extra-pulmonary, atypical, and disseminated forms of
TB were more common. A sizeable number required prolonged courses of ATT, although all improved and prevalence of drug resistance was comparable with the general population.

Although the literature is replete with case reports of TB at odd sites in IIM patients, data on the prevalence of TB in a cohort of IIM in the developing countries is scant. ${ }^{21}$ In a multi-centre study from France published in 2005, of 156 patients with IIM, 18 developed opportunistic infections, of which four had TB. ${ }^{22}$ biochemical findings, and paraclinical features of PM/DM to detect patients at risk of opportunistic infections. Methods The medical records of 156 consecutive PM/DM patients in 3 medical centers were reviewed. Results Eighteen PM/DM patients (11.5\% Another one from Finland, on the contrary, reported a high prevalence of $7.4 \%$ in 176 patients with $1 \mathrm{MM} .{ }^{23}$ Of these, $11(6.25 \%)$ were due to $M$ Tuberculosis, while others were atypical Mycobacteria. A Mexican series of 196 myositis found three cases of TB, all extrapulmonary, which occurred in the inactive phase of myositis though within the first five years of illness. ${ }^{24} \mathrm{~A}$ Taiwanese series of $192 \mathrm{IIM}$ patients reported a prevalence of 3.1\% through six cases. ${ }^{26}$ characteristics and predictors of infections in patients with PM and DM. InMethods. The medical records of 192 PM/DM patients followed up in a tertiary teaching medical centre from 1999 to 2008 were retrospectively reviewed. Seventy-six episodes of major infection, defined as infections requiring $>1$ week of treatment with anti-microbial agents, occurred in 53 (27.6\% On the other hand, a nationwide registry from Taiwan found a prevalence of 1.2\% TB in 4958 newly diagnosed DM patients with a hazard ratio of 2.64 after adjusting for age, gender, and the underlying medical disorder. ${ }^{21}$ It is notable that despite sizeable number of overlap IIM, which frequently can be managed with lower doses of glucocorticoids, the prevalence of TB was higher than all other studies reported. ${ }^{21}$

A higher prevalence of TB in IIM and SLE could be related to the use of immunosuppressive drugs which may lead to activation of latent TB infection (LTBI). Wu PH et al. have established that glucocorticoid and azathioprine use as risk factors for TB in a large nationwide study. ${ }^{21}$ Although rituximab use has been considered safer, Gazaix-Fontaine et al. have described TB even after RTX use, suggesting that background disease, as well as TB prevalence in the country, might be contributively. ${ }^{27}$ This merits further exploration in larger multicentre studies. Intravenous Immunoglobulin could be a safe therapy for the management of active diseases in the setting of TB. ${ }^{28}$ Apart from the activation of latent bacilli, de-novo infection could result from an immunosuppressed state. Both SLE and IIM can, at times, have an associated underlying immunodeficiency, more so in children. ${ }^{29}$ Apart from inherited complement defects, most common being C4 deficiency, seen with both SLE and IIM, polymorphisms 
and copy number variations in $\mathrm{C} 2, \mathrm{C} 4$, and C4A gene have associated with both SLE and polymyositis. (3,31,32 Of particular note, Mannose Binding Lectin (MBL) deficiency is reasonably common and confers a high risk of Tuberculosis. ${ }^{33}$

The higher prevalence of TB in IIM could also be contributed by a failure to clear pooled secretions. Besides, IIM patients often require higher doses of steroids for more extended periods as compared to SLE. Moreover, LTBI is almost universal in the Indian population; hence screening practice for LTBI is not employed in India. ${ }^{34}$ The only study on the use of prevention in 97 SLE patients in India reported a remarkable decline in Tuberculosis from 11.6\% to $2 \%$ over a two-year observation period. ${ }^{35}$ Another paper confirmed that in patients who seroconvert for TST while on biologics, Tuberculosis could be prevented with Isoniazid prophylaxis. ${ }^{36}$ Such initiatives can go a long way in reducing both morbidities, and possibly even mortality. Thus, prevention can be considered in the TB endemic setting in patients receiving high dose corticosteroids like patients with IIM, keeping in mind the challenges from polypharmacy and consequent pill burden, drug interactions, and risk of emergence of resistant strains.

Notably, we found various atypical sites of TB in the current study, paralleling patterns seen in other immunosuppressed states such as HIV. The extrapulmonary TB is common in other rheumatology disease settings such as TNF-inhibitors induced reactivation of TB. ${ }^{37}$ Muscle involvement with TB is infrequent; however, one case of the 24 suggesting a possible preference of the TB bacilli to involve inflamed sites with greater vascularity. Interestingly Nagayama et al. have previously described a case of Tuberculous myo-fasciitis in a patient with DM. ${ }^{38}$ Subsequently, upon review of the eight instances of such Tubercular involvement in literature, three were found to have IIM. ${ }^{38}$ It seems plausible that an inflamed muscle may act as a nidus for infection.

We also noted that a sizeable number of cases developed drug-induced adverse effects. Polypharmacy could complicate the management of TB in IIM. Transaminitis from anti TB treatment can be confused with a relapse of muscle disease, mandating caution while interpreting laboratory tests. With better understanding of the risk, predictors and outcomes of TB in IIM, it may become possible to devise less toxic regimens for management. The strengths of our study include a modest sample size, a comparator group of SLE patients, and follow-up of a subgroup of cases. The limitations are a retrospective nature with the consequent possibility of ascertainment bias as well as under-reporting. There was lack of data on personal history of TB which merits exploration. Further, complete data on immunosuppression received is not available due to retrospective nature of the study. Since the prevalence of TB has gradually changed over the decades with changing treatment practices, disparate time periods of study in the SLE and IIM patients may account for some differences. However, this being the first such study from India assumes relevance as a pilot study. We hope that this exploratory study will pave the way for future prospective studies to understand more about TB in these diseases. The differences in prevalence of TB among various IIM also need to be studied in a prospective study.

Ours being a tertiary care centre receives a referral from more severe illness, and hence prevalence in milder forms of myositis such as amyopathic myositis cannot be ascertained. Besides, the lack of a stringent referral system could have led us to miss cases who reported new symptoms of TB to the general practitioner on follow-up.

\section{CONCLUSION}

Patients with IIM have increased risk of active TB, with common extrapulmonary forms, slower response, and relapses during treatment.

\section{AUTHOR CONTRIBUTION STATEMENT}

$L G, N R$ and $A Z$ were involved in data collection and collation. LG, RA and AA were involved in planning and revising the manuscript critically. All authors approved the final manuscript as submitted and agree to be accountable for all aspects of the work.

\section{CONFLICT OF INTEREST}

The authors declare no conflict of interest.

\section{DISCLOSURES}

Data from this study had been submitted to EULAR 2020 Congress for presentation in an abstract form.

\section{FUNDING}

This study is not funded.

\section{REFERENCES}

1. Muhammed H, Gupta L, Zanwar AA, Misra DP, Lawrence A Agarwal V, et al. Infections Are Leading Cause of In-Hospital Mortality in Indian Patients With Inflammatory Myopathy. J Clin Rheumatol 2019 Dec; 1.

2. 6250311444TB India Report 2018.pdf [Internet]. [cited 2020 May 26]. Available from: https://tbcindia.gov.in/WriteReadData/ I892s/6250311444TB\%20India\%20Report\%202018.pdf

3. Gunn BM, Morrison TE, Whitmore AC, Blevins LK, Hueston L, Fraser RJ, et al. Mannose Binding Lectin Is Required for AlphavirusInduced Arthritis/Myositis. PLoS Pathog 2012 Mar 22;8(3).

4. Denholm JT, McBryde ES, Eisen DP. Mannose-binding lectin and susceptibility to tuberculosis: a meta-analysis. Clin Exp Immunol 2010 Oct;162(1):84-90.

5. Cedzynski M, Szemraj J, Swierzko As, Bak-Romaniszyn L, Banasik M, Zeman K, et al. Mannan-binding lectin insufficiency in children with recurrent infections of the respiratory system. Clin Exp Immunol. 2004 May;136(2):304-11.

6. Bhattacharya PK, Jamil M, Roy A, Talukdar KK. SLE and Tuberculosis: A Case Series and Review of Literature. J Clin Diagn Res JCDR 2017 Feb;11(2):OR01-3. 
7. Feng $\mathrm{PH}$, Tan TH. Tuberculosis in patients with systemic lupus erythematosus. Ann Rheum Dis 1982 Feb 1;41(1):11-4.

8. Bohan A, Peter JB. Polymyositis and Dermatomyositis. N Engl J Med 1975 Feb 13;292(7):344-7.

9. Handbook_on_ICMR_Ethical_Guidelines.pdf [Internet]. [cited 2020 May 26]. Available from: https://naitik.gov.in/DHR/resources/ app_srv/DHR/global/pdf/downloads/Handbook_on_ICMR_ Ethical_Guidelines.pdf

10. Hochberg MC. Updating the American college of rheumatology revised criteria for the classification of systemic lupus erythematosus. Arthritis Rheum 1997;40(9):1725.

11. Wang JY, Lee LN, Hsueh PR, Shih JY, Chang YL, Yang PC, et al. Tuberculous myositis: a rare but existing clinical entity. Rheumatology (Oxford) 2003 Jul;42(7):836-40.

12. 5585665076lndex-TB Guidelines.pdf [Internet]. [cited 2020 Sep 23]. Available from: https://tbcindia.gov.in/WriteReadData/ 1892s/5585665076Index-TB\%20Guidelines.pdf

13. Sharma A, Nagalli S. Chronic Liver Disease [Internet]. StatPearls [Internet]. StatPearls Publishing; 2020 [cited 2020 Sep 20]. Available from: https://www.ncbi.nlm.nih.gov/books/NBK554597/

14. Petri M, Orbai A-M, Alarcón GS, Gordon C, Merrill JT, Fortin PR, et al. Derivation and Validation of Systemic Lupus International Collaborating Clinics Classification Criteria for Systemic Lupus Erythematosus. Arthritis Rheum 2012 Aug;64(8):2677-86.

15. John KJ, Sadiq M, George T, Gunasekaran K, Francis N, Rajadurai E, et al. Clinical and Immunological Profile of Mixed Connective Tissue Disease and a Comparison of Four Diagnostic Criteria. Int J Rheumatol 2020;e9692030.

16. Kahn M-F. Syndrome de Sharp. Syndr Sharp. 1990;40(21):1944-5.

17. 2013 ACR EULAR SSc Classification Criteria_Complete article. pdf [Internet]. [cited $2020 \mathrm{Jul}$ 25]. Available from: https://www. rheumatology.org/Portals/0/Files/2013\%20ACR\%20EULAR\%20 SSc\%20Classification\%20Criteria_Complete\%20article.pdf

18. Le Goff M, Cornec D, Jousse-Joulin S, Guellec D, Costa S, Marhadour T, et al. Comparison of 2002 AECG and 2016 ACR/ EULAR classification criteria and added value of salivary gland ultrasonography in a patient cohort with suspected primary Sjögren's syndrome. Arthritis Res Ther 2017;19:269.

19. Connors GR, Christopher-Stine L, Oddis CV, Danoff SK. Interstitial lung disease associated with the idiopathic inflammatory myopathies: what progress has been made in the past 35 years? Chest 2010 Dec;138(6):1464-74.

20. Bertsias GK, Tektonidou M, Amoura Z, Aringer M, Bajema I, Berden JHM, et al. Joint European League Against Rheumatism and European Renal Association-European Dialysis and Transplant Association (EULAR/ERA-EDTA) recommendations for the management of adult and paediatric lupus nephritis. Ann Rheum Dis 2012 Nov; 71(11):1771-82.

21. Wu P-H, Lin $Y-T$, Yang $Y-H$, Lin $Y-C$, Lin $Y-C$. The increased risk of active tuberculosis disease in patients with dermatomyositis - a nationwide retrospective cohort study. Sci Rep 2015 Nov 17;5: 16303.

22. Marie I, Hachulla E, Chérin P, Hellot M-F, Herson S, Levesque H, et al. Opportunistic infections in polymyositis and dermatomyositis. Arthritis Care Res 2005;53(2):155-65.

23. Airio A, Kinnula V, Kauppi M, Kautiainen H, Hakala M. High association of mycobacterial infections with polymyositis in a non-endemic country for tuberculosis. Ann Rheum Dis 2007 Oct;66(10):1404-5.

24. Vázquez-del Mercado M, Gomez-Bañuelos E, Medrano-Ramírez G, Andrade-Ortega L, Vera-Lastra O, Pizano-Martínez O, et al. Extrapulmonary Tuberculosis Infection in Mexican Patients With Idiopathic Inflammatory Myopathies. J Clin Rheumatol Pract Rep Rheum Musculoskelet Dis 2015 Sep;21(6):331-2.

25. Hernández-Cruz B, Sifuentes-Osornio J, Ponce-de-León Rosales S, Ponce-de-León Garduño A, Díaz-Jouanen E. Mycobacterium tuberculosis infection in patients with systemic rheumatic diseases. A case-series. Clin Exp Rheumatol. 1999 Jun;17(3):289-96.
26. Chen I-J, Tsai W-P, Wu Y-JJ, Luo S-F, Ho H-H, Liou L-B, et al. Infections in polymyositis and dermatomyositis: analysis of 192 cases. Rheumatology 2010 Dec 1;49(12):2429-37.

27. Gazaix-Fontaine E, Ottaviani S, Dieudé P. Pleural tuberculosis under rituximab therapy for anti-synthetase syndrome. Scand $J$ Rheumatol 2018 Jul 4;47(4):338-9.

28. Luzi G, Diamanti AP, Germano V, Laganà B, Di Rosa R, Salemi $S$, et al. Successful treatment with intravenous immunoglobulins in a patient affected by dermatomyositis/systemic lupus erythematosus overlap syndrome and tuberculosis. Clin Immunol 2007 Nov;125(2):127-30.

29. Schmidt RE, Grimbacher B, Witte T. Autoimmunity and primary immunodeficiency: two sides of the same coin? Nat Rev Rheumatol 2018 Jan 1;14(1):7-18.

30. Lintner KE, Patwardhan A, Rider LG, Abdul-Aziz R, Wu YL, Lundström E, et al. Gene Copy-Number Variations (CNVs) of Complement C4 and C4A Deficiency in Genetic Risk and Pathogenesis of Juvenile Dermatomyositis. Ann Rheum Dis 2016 Sep;75(9):1599-606.

31. Ekinci Z, Ozturk K. Systemic lupus erythematosus with C1q deficiency: treatment with fresh frozen plasma. Lupus 2018 Jan 1;27(1):134-8.

32. Jüptner M, Flachsbart F, Caliebe A, Lieb W, Schreiber S, Zeuner $\mathrm{R}$, et al. Low copy numbers of complement $\mathrm{C} 4$ and homozygous deficiency of C4A may predispose to severe disease and earlier disease onset in patients with systemic lupus erythematosus. Lupus 2018 Apr;27(4):600-9.

33. Hristova M, Dourmishev L, Kamenarska Z, Miteva L, Vinkov $A$, Kaneva $R$, et al. MBL2 polymorphisms - manifestations in Bulgarian patients with adult dermatomyositis and systemic lupus erythematosus. Int J Immunogenet 2014;41(2):119-25.

34. Saha S, Kumar A, Saurabh K, Shankar SH, Kashyap A, Nischal N, et al. Current status of treatment of latent tuberculosis infection in India. Indian J Med Sci 2020;71(2):54-9.

35. Trunz BB, Fine P, Dye C. Effect of BCG vaccination on childhood tuberculous meningitis and miliary tuberculosis worldwide: a meta-analysis and assessment of cost-effectiveness. Lancet 2006 Apr;367(9517):1173-80.

36. Suliman S, Geldenhuys H, Johnson JL, Hughes JE, Smit E, Murphy M, et al. Bacillus Calmette-Guérin (BCG) Revaccination of Adults with Latent Mycobacterium tuberculosis Infection Induces Long-Lived BCG-Reactive NK Cell Responses. J Immunol 2016 Aug 15;197(4):1100-10.

37. Borekci S, Duman B, Mazican N, Atahan E, Musellim B, Ongen $\mathrm{G}$, et al. The relationship between smoking habit and tuberculosis in patients under anti-TNF-a treatment. Eur Respir J 2013 Sep 1;42(Suppl 57).

38. Nagayama I, Nagatoya K, Kurahara Y, Mega A, Morita M, Haga $\mathrm{R}$, et al. Tuberculous Fasciitis in Polymyositis: A Rare Case of Extrapulmonary Tuberculosis. Intern Med 2016 Nov 1;55(21):32059. 